\title{
Filling factors and magnetic field strengths of nanoflare-heated coronal active regions: Yohkoh and MDI observations
}

\author{
R. Jain ${ }^{1}$ and S. Yashiro ${ }^{2}$ \\ 1 Department of Physics, UMIST, Manchester M60 1QD, UK \\ e-mail: rjain@suraj.phy.umist.ac.uk \\ 2 Center for Solar Physics and Space Weather, Catholic University of America, Washington, DC 20064, USA \\ e-mail: yashiro@cdaw.gsfc.nasa.gov
}

Received 10 July 2002 / Accepted 26 August 2002

\begin{abstract}
The scaling laws describing the relationship between thermal and magnetic properties of active regions are derived using the concept that solar coronal active regions are heated by numerous small flare-like events (nanoflares). Thus, a coronal active region is viewed as an ensemble of hot elementary filaments created within the coronal magnetic field by random impulsive heating events. The scaling laws obtained are governed by the global energy balance of the active regions and are independent of the details of any heating process (such as the energy of individual heating event or energy spectral index of nanoflares). We examined 61 coronal active regions observed with the soft X-ray telescope aboard Yohkoh and found that such a model yields filling factors (defined as the ratio of the volume of hot plasma to the total volume) in the range 0.002 to 0.015 and magnetic field strengths of $20 \mathrm{G}$ to $40 \mathrm{G}$. The analysis determining the mean magnetic field strength and filling factors of a large number of coronal active regions, observed by Yohkoh, based on nanoflare-heating concept is the first such analysis. We also examine 24 active regions observed with the Michelson Doppler Imager aboard Solar and Heliospheric Observatory and find that the total thermal energy content $E_{\mathrm{th}}$ is related to the total magnetic flux $\phi$ by a power-law index of 1.24 i.e. $E_{\mathrm{th}} \propto \phi^{1.24}$. The thermal pressure $p_{\text {th }}$ of the active regions is related to the magnetic flux density $B_{\mathrm{p}}$ obtained from MDI measurement as: $p_{\text {th }} \propto B_{\mathrm{p}}^{0.5}$.
\end{abstract}

Key words. Sun: activity - Sun: corona - Sun: magnetic fields

\section{Introduction and objective}

Ever since it was discovered that the solar coronal plasma is 1 to 2 million degrees hot, the coronal heating has become one of the most interesting and puzzling problems in astronomy. It is generally accepted that the physical mechanism responsible for heating the solar corona is closely related to the magnetic fields of the corona and the proposed theories include either dissipation of magnetohydrodynamic (MHD) waves or dissipation of stressed magnetic field. However, the conversion of magnetic energy to heat occurs on spatial scales that are far smaller than the resolution achieved by the present-day solar instruments. Therefore, in the absence of any direct observational evidence, it has proved difficult to determine the exact cause of the heating and we are having to rely more on indirect methods to identify the mechanism which is likely to heat and maintain the solar corona to few million degrees.

One of the recent and popular approaches to the coronal heating problem is to derive, observationally and theoretically, the scaling relationship between thermal and magnetic parameters of solar coronal loops or active regions. For example,

Send offprint requests to: $\mathrm{R}$. Jain,

e-mail: rjain@suraj .phy.umist.ac.uk
Golub et al. (1980) found that the coronal thermal energy, $E_{\mathrm{th}}$ and the photospheric magnetic flux $\phi$, obey power-law relationship $E_{\mathrm{th}} \propto \phi^{1.5}$. They also found that the gas pressure $p$ and the magnetic flux density $B$ obey power-law index of 1.6. Fisher et al. (1998) found that the X-ray luminosity of an active region scales almost linearly with the total magnetic flux. The well-known RTV (Rosner et al. 1978) scaling for the observed gas pressure $p$, loop length $L$, temperature $T$ and the heating rate $E_{\mathrm{H}}$ is given by

$$
T \approx 1.4 \times 10^{3}(p L)^{1 / 3}, E_{\mathrm{H}} \approx 10^{5} p^{7 / 6} L^{-5 / 6} .
$$

Similarly other models predict that the heating rate has a power-law dependence on the magnetic field strength and the size of the region. It is now believed that establishing the relation between magnetic and thermal properties of active regions may be vital in understanding the coronal heating process. Coronal heating requires

$E_{\mathrm{H}} \propto B^{\alpha} L^{\beta}$

where $\alpha$ and $\beta$ are indices that are different for different heating models. Thus, the observed correlations (e.g. Yashiro $\&$ Shibata 2001) provide a valuable test for coronal heating theories. 
In this context, it should be noted that the coronal magnetic field strength $B$ is a key physical parameter in many of the scaling laws of the coronal active regions. However, determining its absolute value has been a challenge for observers. A commonly used direct method involves analysing the EUV coronal emission spectrum which is modified due to Zeeman effect. The methods based on gyroresonant emission (e.g. Lee et al. 1999) can detect magnetic field strengths of few hundred Gauss but it has a rather low spatial resolution (5 arcsec or larger).

Indirect methods for determining coronal magnetic field include extrapolation of field lines using photospheric magnetograms and assuming potential or force-free fields but such models have ambiguities. Recently, Nakariakov \& Ofman (2001) have used MHD oscillations in the coronal loop to determine the magnetic field strength. However, they report that errors in the measurement of period of oscillation and loop length and assumptions about the density inside and outside the loop can lead to errors in the magnetic field strength by as much as $30 \%$.

The other key parameter is the filling factor $f$ which is defined as the fraction of the total volume filled with hot $\mathrm{X}$-ray emitting plasma. The density of a coronal loop is usually computed from the total emission measure $E M$ by using the expression:

$n=\left(\frac{E M}{f V}\right)^{1 / 2}$

where $V$ is the volume. The presence of $f$ in the above expression suggests that the observed radiation may be coming from few localised blobs of plasma rather than uniformly distributed (along the line-of-sight) plasma. Most studies assume loops to be uniformly filled and take $f=1$. However, this may be a wrong assumption (see e.g. Porter \& Klimchuk 1995). Theoretically, the filling factor for hot X-ray emitting plasma from the spatial discreteness of nanoflare heating is predicted to be less than unity (see e.g. Cargill \& Klimchuk 1997; Vekstein \& Katsukawa 2000). Observationally, it can be obtained by comparing the actual density of emitting plasma (from spectral lines) to the mean plasma density derived from the detected emission measure. However, the results are quite controversial and its study, still quite premature.

The general aim of this paper is to provide scaling laws relating the temperature, filling factor, thermal energy, emission measure etc. to the magnetic field strength and length of the active regions. A specific objective is to use these scaling laws to determine the mean magnetic field strengths and filling factors of 61 active regions whose temperature and emission measure are obtained using Yohkoh data. How the total thermal content of the coronal active regions is related to the total magnetic flux observed by Michelson Doppler Interferometer (MDI) at the photospheric level will also be explored briefly.

The present study is motivated by the theoretical model of Vekstein \& Katsukawa (2000) (hereafter referred to as V\&K) and recent observations reported by Yashiro \& Shibata (2001) (hereafter referred to as $\mathrm{Y} \& S$ ). V\&K derived scaling laws for the nanoflare heating, which relate integral characteristics of coronal loops (such as temperature, emission measure, filling factor, etc.) with the size of the region, the strength of the coronal magnetic field, and the heating flux $q$. Y\&S examined the properties of 64 active regions observed with Yohkoh and found that the scaling law between gas pressure $p$, magnetic flux density $B$, and active region size $L, p \propto B^{0.78} L^{-0.16}$, is consistent with that of steady coronal loops. V\&K concluded that the coronal filling factor is likely to be small and their scaling laws fit well with some Yohkoh observations (see also, Cargill \& Klimchuk 1997; Jain \& Vekstein 2002) whereas in Y\&S, the filling factor was assumed to be unity. We consider V\&K model and rewrite the scaling laws using a different expression for the radiative time-scale, which is more relevant for the active regions examined by Y\&S. We then, determine the mean magnetic field strength and filling factor for the active regions reported in $\mathrm{Y} \& \mathrm{~S}$ using the newly derived scaling laws.

The paper is organised as follows. In Sect. 2, we briefly discuss the formation and thermal evolution of the hot filaments. Scaling laws for thermal and magnetic parameters (plasma pressure, thermal energy, magnetic field strength, filling factor etc.) are derived for active regions which are viewed as superposition of many hot filaments. In Sect. 3, we briefly describe the observational data and show the results obtained by using the scaling laws between thermal and magnetic properties. We compare our results with other similar studies in Sect. 4. We then summarise and conclude in Sect. 5.

\section{The model and scaling laws}

We assume that coronal active region consists of large number of hot magnetic filaments. Since the processes that release energy in the corona (e.g. magnetic reconnection or resonant wave absorption) are believed to be very fast compared with the typical lifetime of hot filaments, it is reasonable to assume the heating event to be instantaneous. Thus, we assume that these filaments are created by randomly distributed impulsive heating events referred to as "nanoflares". In our context "nanoflares" means rapid and localised heat deposition regardless of its particular mechanism. We follow the self-consistent model of $\mathrm{V} \& \mathrm{~K}$ for the creation and thermal evolution of the filaments. In brief, the magnetic field of an active region and the amount of the heat input define the size of the filaments. The thermal pressure inside the filament is assumed to be in equilibrium with the magnetic pressure outside it. The thermal evolution of the filament is determined by interaction of its strong heat flux with the low lying dense chromosphere.

Following V\&K, the filament cooling can be mainly described by two phases. During the first phase, the total thermal energy in the filament is conserved as the downward conductive heat flux is balanced by the evaporative upflow of enthalpy. Thus, the radiation losses are neglected. The second stage is dominated by radiation losses. The total lifetime $\tau_{\mathrm{h}}$ of a hot filament is determined by losses due to thermal conductivity and radiation. Assuming the conductive $\left(\tau_{\mathrm{c}}\right)$ and radiative $\left(\tau_{\mathrm{r}}\right)$ time-scales (Cargill \& Klimchuk 1997) as

$$
\begin{aligned}
\tau_{\mathrm{c}} & =\frac{4 \times 10^{-10} n L^{2}}{T^{5 / 2}} \\
\tau_{\mathrm{r}} & =\frac{1.17 \times 10^{-3} T^{5 / 2}}{n} ; \quad 1.5 \times 10^{6}<T<3.55 \times 10^{6} \mathrm{~K},
\end{aligned}
$$


one can estimate the lifetime $\tau_{\mathrm{h}}$ of hot filament during which it contributes to the $\mathrm{X}$-ray luminosity of the region, as (see V\&K)

$\tau_{\mathrm{h}}(\mathrm{sec})=1.34 \times 10^{-5} L_{\mathrm{c}}$,

where $L_{\mathrm{c}}$ is the size of the active region. Note that the expression (2) for $\tau_{\mathrm{r}}$ is different from the one used in $\mathrm{V} \& \mathrm{~K}$ (see Eq. (6) in V\&K). Hot filaments in an active region are at different phases of cooling but general characteristics of the active regions are not expected to be sensitive to the details of the temperature distribution of filaments. It is assumed that each hot filament spends most of its lifetime in the phase when the transition from the conductive to radiative cooling occurs (see $\mathrm{V} \& \mathrm{~K})$. Thus, the mean temperature $T_{\mathrm{c}}$ of such coronal active regions when observed with broadband X-ray telescope (e.g. Yohkoh) should be close to the transition temperature and is related to the mean magnetic field strength $B_{\mathrm{c}}$ and the size, $L_{\mathrm{c}}$ of the active region. Thus, equating (1) and (2) and using the pressure balance inside and outside the filament yields, for $1.5 \times 10^{6}<T_{\mathrm{c}}<3.55 \times 10^{6} \mathrm{~K}$ :

$T_{\mathrm{c}} \approx 6.8 \times 10^{2} B_{\mathrm{c}}^{4 / 7} L_{\mathrm{c}}^{2 / 7}$.

By knowing the mean temperature $T_{\mathrm{c}}$ and the size, $L_{\mathrm{c}}$, of the active region from observations, one can estimate the mean magnetic field strength of the active regions by using Eq. (4). The plasma density $n_{\mathrm{c}}$ that corresponds to the above temperature $T_{\mathrm{c}}$ is given by

$n_{\mathrm{c}}=2 \times 10^{11} B_{\mathrm{c}}^{10 / 7} L_{\mathrm{c}}^{-2 / 7}$.

Note that the plasma pressure $p_{\mathrm{c}}\left(=2 n_{\mathrm{c}} k T_{\mathrm{c}}\right)$ in the filament is balanced by the magnetic pressure $p_{\mathrm{M}}\left(=B_{\mathrm{c}}^{2} / 8 \pi\right)$ outside it. The total emission measure is given by

$(E M)_{\mathrm{t}}=n_{\mathrm{c}}^{2} f V$,

where $V$ is the volume of the active region; $f$, the filling factor is the fraction of the total volume of the active region containing X-ray emitting plasma and can be estimated as follows.

If the heat flux $q\left(\mathrm{erg} \mathrm{cm}^{-2} \mathrm{~s}^{-1}\right)$, required to maintain a steady state hot corona, is provided by nanoflares each with energy $\Delta E$, the occurrence rate of nanoflares per unit area is $q / \Delta E$. Thus, if the active region has cross-sectional area $A$, the average number of nanoflares per unit time is $q A / \Delta E$. Since each nanoflare creates a hot filament with lifetime, which is the sum of the conductive and radiative time intervals (V\&K), the total number of such filaments present within an active region at any given time is (using Eq. (3))

$N=\frac{q A}{\Delta E} \times 1.34 \times 10^{-5} L_{\mathrm{c}}$.

From an energy conservation condition, the cross-section area $\Delta A$, of the filament can be given by $(\mathrm{V} \& \mathrm{~K})$

$\Delta A=\frac{16 \pi}{5} \frac{\Delta E}{B_{\mathrm{c}}^{2} L_{\mathrm{c}}}$.

Therefore, the filling factor of the active region can be estimated as

$f=N \frac{\Delta A}{A} \approx 1.35 \times 10^{-4} q B_{\mathrm{c}}^{-2}$.
Since the filling factor is of the same order as the average value of $\beta$ (the ratio of the thermal pressure $p_{\text {th }}$ to the magnetic pressure $p_{\mathrm{M}}=\frac{B^{2}}{8 \pi}$ ), the total thermal energy $E_{\mathrm{th}}$ can be given by

$E_{\text {th }}=\left(\frac{3}{2}\right) p_{\text {th }} V=\frac{3}{2} \frac{B_{\mathrm{c}}^{2}}{8 \pi} f V$.

Equations (4)-(10) provide scaling laws for thermal and magnetic properties of nanoflare-heated active region and can be used for estimating important quantities like the mean magnetic field strength and filling factors of X-ray active regions by using the observed values of temperature, size and total emission measure of such regions.

\section{Observational data and analysis}

We use the derived temperatures and emission measures of 61 active regions reported in $Y \& S$. The mean temperature of these regions range from 1.7 to $3.5 \mathrm{MK}$. The active regions were observed by Yohkoh/SXT using Al and AlMg filters (see Y\&S for details). For the sake of simplicity, we assume a single temperature across each active region. Ideally an active region has a multi-temperature structure, but in the present study we will not consider the spatial variations of temperature within a single active region. We integrate the soft X-ray emission over the entire region, and calculate the filter-ratio of thin $\mathrm{Al}$ and $\mathrm{AlMg}$ filters. Thus, the measured temperature is a mean temperature weighted with the soft X-ray brightness. Because these filters have the highest sensitivity at $5 \mathrm{MK}$ plasma, the brightest part in an SXT image is hottest (if the temperature is less than $5 \mathrm{MK})$. Thus, the measured temperature in this study is close to the maximum temperature of active regions. We do not take into account the effect of the hydrostatic weighting bias (see Aschwanden \& Nitta 2000) because (1) we have analysed the brightest part of active regions and (2) we do not consider the temperature profile along the coronal loops. Each active-region is assumed to be a hemisphere. Expressing quantities in terms of the observed region size $L_{\mathrm{c}}$ and following $\mathrm{Y} \& \mathrm{~S}$, assumptions made include volume $V=\frac{2}{3} \pi\left(\frac{L_{\mathrm{c}}}{2}\right)^{3}$. Figure 1a plots the temperature $T_{\mathrm{c}}$ and region size $L_{\mathrm{c}}$ of the 61 active regions. Using the least squares method applied logarithmically, we can describe the relationship as:

$T_{\mathrm{c}} \propto L_{\mathrm{c}}^{0.26 \pm 0.08}$

where the $3 \sigma$ error of the power-law index is shown.

\subsection{Determination of magnetic field strengths and filling factors}

The observed temperature $T_{\mathrm{c}}$ and the size $L_{\mathrm{c}}$ are used in Eq. (4) to obtain the magnetic field strength $B_{\mathrm{c}}$. Figure $1 \mathrm{~b}$ plots $B_{\mathrm{c}}$ versus $L_{\mathrm{c}}$. Although the solid line denotes a relationship between these two quantities as $B_{\mathrm{c}} \propto L_{\mathrm{c}}^{-0.04 \pm 0.05}$, it can be seen clearly from the figure that the data points are quite scattered and there is no obvious empirical scaling law between $B_{\mathrm{c}}$ and $L_{\mathrm{c}}$.

Substituting the expressions for $n_{\mathrm{c}}$ and $f$ from Eqs. (5) and (9), into Eq. (6) yields

$(E M)_{\mathrm{t}} \approx 1.4 \times 10^{18} q B_{\mathrm{c}}^{6 / 7} L_{\mathrm{c}}^{17 / 7}$. 

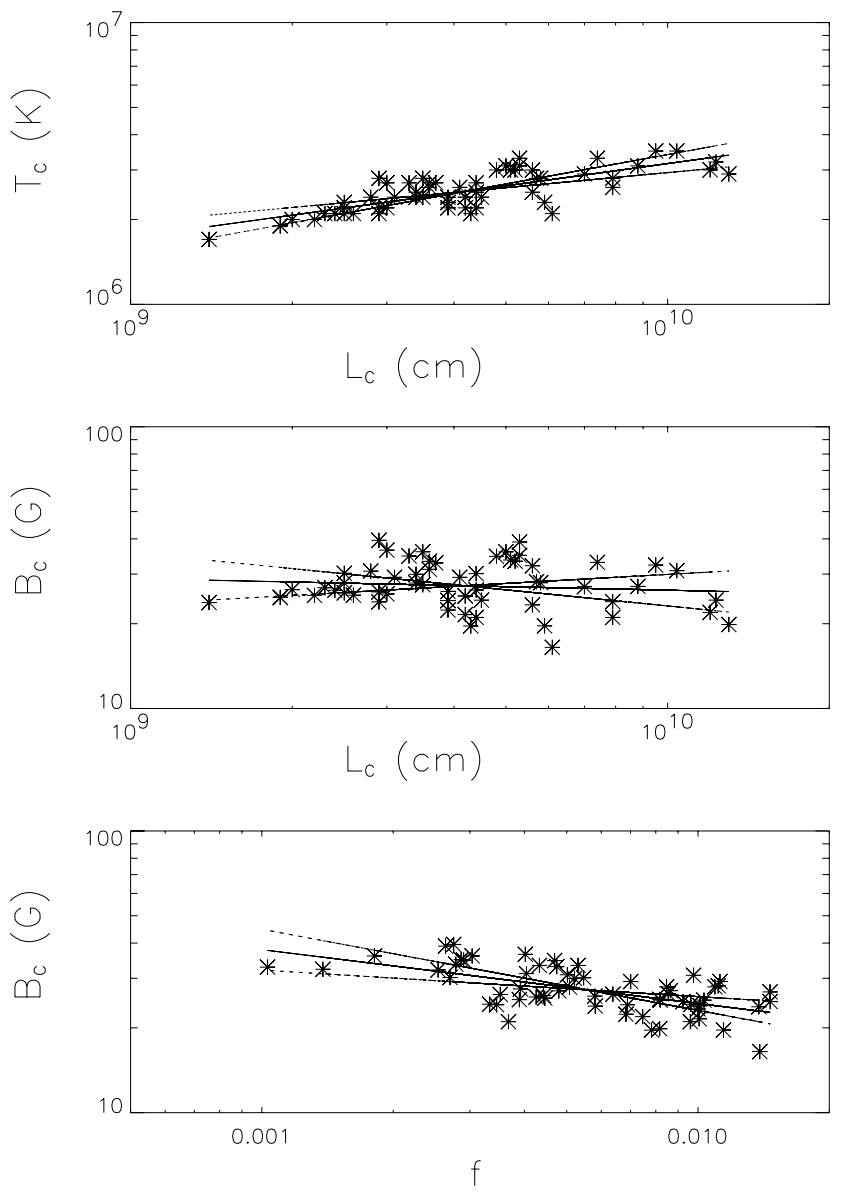

Fig. 1. Empirical scaling laws linking the temperature $T_{\mathrm{c}}$, the magnetic field strength $B_{\mathrm{c}}$, the region size $L_{\mathrm{c}}$, the filling factor $f$ of the 61 coronal active regions. Solid lines denote the least-squares fit a) $T_{\mathrm{c}} \propto L_{\mathrm{c}}^{0.26}$ b) $B_{\mathrm{c}} \propto L_{\mathrm{c}}^{-0.04}$ c) $B_{\mathrm{c}} \propto f^{-0.19}$. The dashed lines show the $3 \sigma$ error of the slope.

By knowing $B_{\mathrm{c}}$ and the observed value of total emission measure, one can then use Eq. (12) to determine the heat flux $q$ needed to provide the observed emission. Finally, the filling factor $f$ can be obtained from Eq. (9). Figure 1c plots $B_{\mathrm{c}}$ versus $f$. Using the least squares method, we obtain the following empirical scaling law (see the solid line in Fig. 1c):

$B_{\mathrm{c}} \propto f^{-0.2 \pm 0.03}$.

\subsection{Magnetic and thermal properties}

We obtain the pressure $p_{\text {th }}\left(=\frac{B_{\mathrm{c}}^{2}}{8 \pi} f\right)$ which is the mean value for the active regions. Figure 2 shows the mean pressure $p_{\text {th }}\left(\mathrm{erg} \mathrm{cm}^{-3}\right)$ as a function of region length $L_{\mathrm{c}}(\mathrm{cm})$ of the 61 active-regions. As can be seen, there is lot of scatter in the data points and there is no clear empirical scaling law for these quantities.

We now consider those active regions (24 of the 61) whose magnetograms were obtained around the same time as their X-ray observations by Yohkoh. Thus, we examine the total magnetic flux $\phi$ of 24 active regions measured by Michelson Doppler Imager (MDI) aboard Solar and

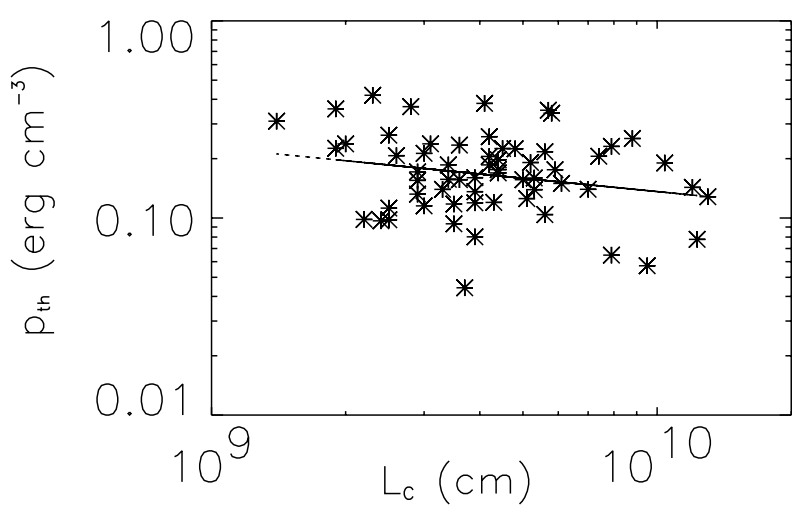

Fig. 2. The coronal gas pressure $p_{\mathrm{th}}$ as a function of the region size $L_{\mathrm{c}}$. It is apparent that there is lot of scatter in the data points and there is no clear relationship between these quantities. However, using leastsquares method, a rough estimate for $p_{\text {th }}$ and $L_{\mathrm{c}}$ can be given by $p_{\text {th }} \propto$ $L_{\mathrm{c}}^{-0.22}$ and is shown by the dashed line.

Heliospheric Observatory (SOHO) (see Scherrer et al. 1995). Recall that MDI uses the Ni $6768 \AA$ photospheric absorption line which is believed to be formed at about $200 \mathrm{~km}$ above the temperature minimum whereas Yohkoh observations are made in coronal regions which are at least few thousand $\mathrm{km}$ above the temperature minimum region. The realtionship between the derived values of the total thermal energy $E_{\text {th }}$ (see Eq. (10)) and the total magnetic flux $\phi$ of the above mentioned 24 active regions is shown in Fig. 3a. It can be estimated as:

$E_{\mathrm{th}} \propto \phi^{1.24 \pm 0.08}$.

Note that for these 24 active regions, the total thermal energy $U_{\text {th }}$ obtained in $\mathrm{Y} \& \mathrm{~S}$ ranges from $1.2 \times 10^{28}$ erg to $2.5 \times 10^{30}$ erg whereas in the present study, the total thermal energy content $E_{\text {th }}$ varies from $4 \times 10^{26} \mathrm{erg}$ to $10^{29} \mathrm{erg}$. The reason for this difference, as can be seen from Eq. (10), is the filling factor $f$. In $\mathrm{Y} \& \mathrm{~S}$, the filling factor was assumed to be unity. We can relate the two thermal energies as: $E_{\mathrm{th}}=U_{\mathrm{th}} f$.

The region size $L_{\mathrm{p}}$ and the total magnetic flux $\phi$ can be used to estimate the mean magnetic field strength $B_{\mathrm{p}}$ of these regions at the photospheric level:

$B_{\mathrm{p}}=\frac{\phi}{L_{\mathrm{p}}^{2}}$.

Thus, $B_{\mathrm{p}}$ calculated using the above expression ranges from $42 \mathrm{G}$ to $290 \mathrm{G}$. Figure $3 \mathrm{~b}$ shows the plot of mean plasma pressure $p_{\text {th }}$ of the coronal active regions versus their magnetic flux density $B_{\mathrm{p}}$. The solid line indicates the relationship:

$p_{\text {th }} \propto B_{\mathrm{p}}^{0.46 \pm 0.13}$.

Exactly how the magnetic fields expand from photospheric flux tube to fill the corona is not known. However, some rough estimates can be obtained by simple argument of flux conservation. Thus,

$\phi=B_{\mathrm{c}} r^{2}$

where $r>L_{\mathrm{c}}$. Hence, the magnetic filling factor, $f_{B}=\left(\frac{L_{\mathrm{c}}}{r}\right)^{2}$ can be obtained by knowing $r$ from (17) and knowing the observed 

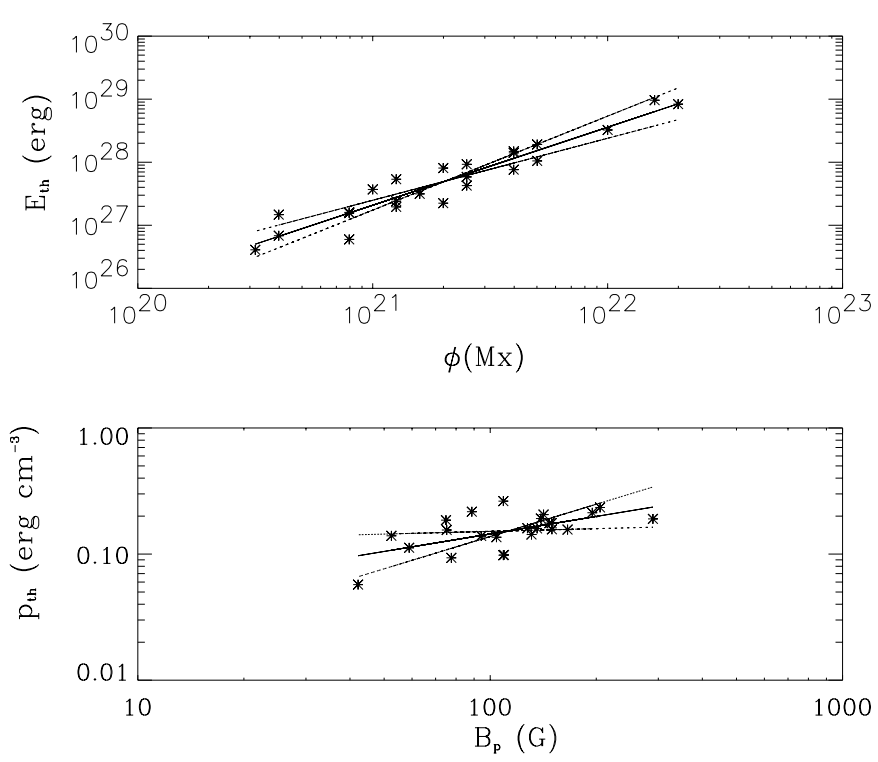

Fig. 3. a) The total thermal energy content $E_{\text {th }}(\mathrm{erg})$ as derived from Eq. (10) as a function of the total magnetic flux $\phi(\mathrm{Mx})$ of the $24 \mathrm{ac}-$ tive regions observed by MDI. The solid line shows $E_{\mathrm{th}} \propto \phi^{1.24}$. The dashed line show the $3 \sigma$ error of the slope. b) The plasma pressure $p_{\text {th }}\left(\mathrm{erg} \mathrm{cm}^{-3}\right)$ versus the magnetic flux density $B_{\mathrm{p}}(\mathrm{G})$ measured from MDI. The solid line indicates $p_{\text {th }} \propto B_{\mathrm{p}}^{0.46}$. The dashed line show the $3 \sigma$ error of the slope.

coronal active region size $L_{\mathrm{c}}$. It is assuring to know that the magnetic filling factor $f_{B}$ thus obtained for the 24 active regions are less than unity.

\section{Comparison with previous studies}

Determining the scaling laws between thermal and magnetic properties of coronal active region is vital to the coronal heating problem. The power-law exponents deduced from theory and observations give important clues about the physical mechanism responsible for heating the coronal active regions. Let us briefly highlight some such studies.

Since an active region consists of many loops, we can compare the scaling laws of active regions with those of coronal loops. Many observational studies have investigated scaling laws of the form:

$p \propto B^{\alpha}, p \propto L^{\beta}, T \propto L^{\gamma}, B \propto L^{\delta}$.

Klimchuk \& Porter (1995) studied steady coronal loops from Yohkoh data and found a power-law index of $-1.82 \leq \beta \leq$ -0.22 . They also reported that the temperature of a loop is not dependent on its size, suggesting $\gamma=0$. They did not examine the magnetic properties of the loops but when Mandrini et al. (2000) considered the same loops which were analysed by Klimchuk and Porter, they found that for loop lengths $50<L<300 \mathrm{Mm}$, the power-law index $\delta=-0.97 \pm$ 0.25 . The results of Klimchuk and Porter study suggests that radiative losses are comparable with conductive losses for soft X-ray (SXR) loops observed by Yohkoh.

Aschwanden et al. (1999) considered loops observed with Extreme-Ultaviolet Imaging Telescope (EIT) and found $\beta=-0.41 \pm 0.12, \gamma=0$ and $\delta=-1.02 \pm 0.25$. Their powerlaw index $\delta$ is similar to the result of Mandrini et al. (2000). However, Aschwanden et al. (1999) argue that since the EUV loops observed by EIT have lower temperature than SXR loops of Yohkoh, the radiative loss is dominant compared with conductive loss.

Y\&S (2001) studied active regions using Yohkoh data and found $\alpha=0.78 \pm 0.23, \beta=-0.16 \pm 0.21, \gamma=0.28 \pm 0.08$ and $\delta=0$. They concluded that their scaling laws are consistent with that of steady coronal loops such as those found by Klimchuk \& Porter (1995).

In this study we have considered the radiative loss to be comparable with the conductive loss and derived theoretically, the scaling laws for coronal active regions by assuming that these regions consist of numerous hot filaments whose length is determined by the coronal magnetic field. Note that the scaling laws derived in this study reflect the statistical steady state of active regions consisting of numerous time-dependent filaments rather than a steady state thermal equilibrium of a single loop. We used the Yohkoh data reported in Y\&S (2001) and found $\alpha=0.46 \pm 0.13, \beta=-0.22, \gamma=0.26 \pm 0.08$ and $\delta=-0.04 \pm 0.05$. Unlike the above mentioned studies where the filling factor $f$ is assumed to be unity, we found from our model that the filling factor of the coronal active regions is much less than unity.

Let us now consider various observational requirements for coronal heating mechanisms. The relationship between the heating flux $F$, magnetic flux density $B$ and loop length $L$ is: $F \propto B^{a} L^{b}$ where the values of $a$ and $b$ are different for different heating mechanisms.

Theoretically, Galsgaard \& Nordlund (1996) obtained $a=$ $2, b=-1$ for their magnetic reconnection model. Others (Longcope 1996; Fisher et al. 1998; Sturrock et al. 1999) have suggested $a \approx 1$.

Observationally, Golub et al. (1980) suggested $a \approx 2$. The scaling laws derived in Y\&S from Yohkoh observations would suggest $a=0.91 \pm 0.27$ and $b=-0.02 \pm 0.25$. Y\&S report that their results are consistent with Alfvén model (see Y\&S 2001) if the filling factor $f$ and the footpoint velocity $v_{\text {ph }}$ are constant and also consistent with the chromospheric reconnection model of Sturrock et al. (1999) for strong fields.

In the present study, our analytical predictions when applied to the same Yohkoh data as in Y\&S, yield $a \approx 0.54 \pm 0.15$ and $b \approx-0.09$. This value of $a$ is significantly lower than any previously reported values. It may be that despite the advantage that we are able to determine filling factors and mean magnetic field strengths of coronal active regions, our model needs further refinements in order to probe the details of heating (e.g. energy distribution of nanoflares).

\section{Conclusions}

In this paper, we considered a nanoflare heating model for coronal active regions and analysed various scaling laws to obtain thermal and magnetic properties. These scaling laws depend on the heat flux $q$ but otherwise are independent of any details of the heating process e.g. the energy distribution of nanoflares. We investigated various observations of coronal active regions 
by Yohkoh and determined the mean magnetic field strength $B_{\mathrm{c}}$ of these active regions by considering that the active regions in the solar corona are heated by many small flare-like events known as "nanoflares". It should be noted that the mean magnetic field strengths for the coronal active-regions obtained in this study only offer a rough guide and one cannot rule out other models and ways to obtain the same. Nevertheless, we have shown a unique way to combine a nanoflare heating model with observations to determine the mean magnetic field strength of the coronal active regions. We also found that the filling factors range from 0.002 to 0.015 . The determination of the mean magnetic field strength and filling factors of a large number of active regions, observed by Yohkoh, based on the nanoflareheating concept is the first such study. We examined the observations made by MDI to relate the magnetic and thermal properties of the active regions. A better picture could be envisaged for the magnetic structure of these regions from improved coronal extrapolation of photospheric vector magnetograms.

Finally, in this study we have determined the gas pressure and coronal magnetic field strengths for active regions, and so the plasma beta, $\beta$, can be predicted for these active regions. It would be interesting and important to check this observationally. However, this would require measuring the coronal magnetic field strengths independently and at the moment there is no such observation except for those of flare loops during the decay phase (determined by radio observations). We believe that an alternative way could be to extrapolate from magnetic field observations at the photosphere and/or chromosphere (for example, with the assumption of force-free field).
Acknowledgements. This work was supported by the UK Particle Physics and Astronomy Research Council. RJ would also like to thank Drs. G. Vekstein and Y. Katsukawa for many useful discussions. We also appreciate the referee for a very helpful and precise report.

\section{References}

Aschwanden, M. J., Tarbell, T. D., Nightingale, R. W., et al. 2000, ApJ, 535, 1047

Aschwanden, M. J., Nitta, N. 2000, ApJ, 535, L59

Cargill, P. J., \& Klimchuk, J. A. 1997, ApJ, 478, 799

Fisher, G. H., Longcope, D. W., Metcalf, T. R., \& Pevtsov, A. A. 1998 , ApJ, 508, 885

Golub, L., Maxson, C., Rosner, R., Vaiana, G. S., \& Serio, S. 1980, ApJ, 238, 343

Galsgaard, K., \& Nordlund, A. 1996, J. Geophys. Res., 101, 113445

Jain, R., \& Vekstein, G. 2002, A\&A, submitted

Klimchuk, J. A., \& Porter, L. J. 1995, Nature, 377, 131

Lee, J., White, S. M., Kundu, M. R., Mikić, Z., \& McClymont, A. N. 1999, ApJ, 510, 413

Longcope, D. W. 1996, Sol. Phys., 169, 91

Mandrini, C. H., Demoulin, P., \& Klimchuk, J. A. 2000, ApJ, 530, 999

Nakariakov, V. M., \& Ofman, L. 2001, A\&A, 372, L53

Porter, L. J., \& Klimchuk, J. A. 1995, ApJ, 454, 499

Rosner, R., Tucker, W. H., \& Vaiana, G. S. 1978, ApJ, 220, 643

Scherrer, P. H., Bogart, R. S., Bush, R. I., et al. (and the MDI Engineering team) 1995, Sol. Phys., 162, 129

Sturrock, P. A., Roald, C. B., \& Wolfson, R. 1999, ApJ, 524, L75

Vekstein, G., \& Katsukawa, Y. 2000, ApJ, 541, 1096

Yashiro, S., \& Shibata, K. 2001, ApJ, 550, L113 